\section{TCH-017 EYE DROPS MADE FROM PLATELET-RICH PLASMA: DEVELOPMENT AND USE OF A NEW MASTER FORMULA}

doi:10.1136/ejhpharm-2013-000276.208

R Romero Domínquez, A Gómez Sánchez, J Arenas Villafranca, C López Martin, ME Blanco Rivas, S Santana Martínez, I Escudero Santos, V Faus Felipe. Hospital Costa del Sol, Pharmacy and Nutrition, Marbella, Spain

Background Eye drops made from platelet-rich plasma are used in the treatment of ocular surface dysfunctions after LASIK refractive surgery, severe dry eye and corneal ulcers.

Purpose To describe the process of enriching the plasma, and the dosage used.

Materials and Methods This master formula was developed from the information obtained from a literature search in PubMed and Embase.

Results We extracted blood in $10 \mathrm{ml}$ tubes containing 3.2\% sodium citrate. The amount required depends on the length of treatment. From each $10 \mathrm{ml}$ of blood processed, we obtained 3-4 $\mathrm{ml}$ of plateletrich plasma, sufficient for one week of treatment. We received 10 tubes of blood from each patient. These were centrifuged at a speed of $1400 \mathrm{rpm}$ for 10 minutes to obtain maximum concentration. The tubes were kept in an upright position, to avoid mixing the contents and waste. The eye drops were prepared in sterile conditions in a laminar flow cabinet. Using a sterile Pasteur pipette, we removed the top layer of the centrifuged blood which is the plateletrich plasma. The plasma was collected in syringes and was then stored in sterile light-resistant containers, each containing 2-3 ml of platelet-rich plasma. It remained stable for a week in a fridge, or 3 months in a freezer. Patients were treated with a dose of 5-10 drops per day. The duration varied according to the diagnosis (between 1 week and 1 month of treatment).

Conclusions Platelet-rich plasma eye drops made in the Pharmacy Service after consulting previously published research and according to Royal Decree 1751/2001 are a new and alternative treatment for corneal ulcers, dry eye and post-LASIK dysfunction of the ocular surface

No conflict of interest.

\section{TCH-018 FORMULATION OF AN ORAL SOLUTION CONTAINING "POTION JOULIË" PHOSPHORUS TO COUNTERACT THE SHORTAGE OF PHOSPHONEUROS}

doi:10.1136/ejhpharm-2013-000276.209

${ }^{1} \mathrm{~L}$ Pailhas, ${ }^{1} \mathrm{C}$ Therasse, ${ }^{1} \mathrm{~A}$ Papon, ${ }^{2} \mathrm{~F}$ Brion, ${ }^{1 T}$ Storme. ${ }^{1}$ Hopital Robert Debre (Ap-Hp), Pharmacy, Paris, France; ${ }^{2}$ Hopital Robert Debre (Ap-Hp) Université Paris Descartes Faculté De Pharmacie, Pharmacy, Paris, France

Background In October 2011 a shortage of Phosphoneuros became apparent. This oral solution containing phosphorus is prescribed in the treatment of diseases where phosphorus intake is essential. None of the available drugs was suitable for paediatric needs. Literature searches were conducted to identify formulas in national formularies and pharmacopoeias or already developed in other compounding hospitals, but no consensus 'Potion Joulié' formula was found.

Purpose To provide patients with a concentrated oral solution of phosphorus. A feasibility study was performed. The aim was to give an expiry date of 3 months.

Materials and Methods A batch of 3 bottles was produced. Visual appearance, $\mathrm{pH}$, phosphorus and sodium contents were determined. At M0, M1 and M3 a microbiological assay was performed according to the Ph Eur (5.1.4).

Results The formula we adopted consists of (for $100 \mathrm{ml}$ ): $20.40 \mathrm{~g}$ of phosphoric acid 50 per cent, $16.5 \mathrm{~g}$ of sodium phosphate dibasic anhydrous, $50 \mathrm{~mL}$ of sterile water and simple syrup. The phosphorus strength of $67.4 \mathrm{mg} / \mathrm{mL}$ is close to that of Phosphoneuro. For 12 weeks, the solution appeared unchanged, clear and colourless. $\mathrm{pH}$ about 4.14 remained constant. Sodium and phosphorus contents were stable and the observed values were within $10 \%$ of the theoretical values. Microbiological results were in accordance with European Pharmacopeia: viable aerobic bacteria $\leq 10^{3}(\mathrm{CFU} / \mathrm{ml})$, fungal $\leq 10^{2}$, no E.coli.

Conclusions Microbiological compliance and physicochemical stability were verified at 12 weeks according to the standards of the European Pharmacopeia. After users had insisted, the French Regulatory agency urged Bouchara Recordati to produce Phosphoneuros again, effective in May 2012. This is an example of the hospital pharmacist's role in compounding drugs to allow patients to continue their treatment in case of shortages of commercial products.

No conflict of interest.

\section{TCH-019 IMPLEMENTATION OF A MONITORED INFORMATION SYSTEM PROTOCOL IN PHARMACEUTICAL MONITORING}

doi:10.1136/ejhpharm-2013-000276.210

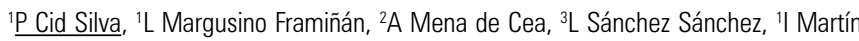
Herranz. 'Complejo Hospitalario Universitario A Coruña, Department of Pharmacy, A Coruña, Spain; ${ }^{2}$ Complejo Hospitalario Universitario A Coruña, Internal Medicine Service, A Coruña, Spain; ${ }^{3}$ Complejo Hospitalario Universitario A Coruña, R\&D Department of Computing Service, A Coruña, Spain

Background Information and communication technologies increase efficiency and safety in health systems. The SiMON protocol (Monitored Information System), developed by the R\&D department of hospital's Computing Service, provides a tool for monitoring patients attending a particular consultation and is adaptable in line with the needs of each clinical service. Its main objective is to streamline care by automating patient information related to such consultation. Furthermore, it provides a record for future analysis of information collected, making it possible to export information, scorecards and predict comorbidities.

Purpose To describe the implementation of SiMON in pharmaceutical monitoring of patients with viral diseases (HIV/HCV).

Materials and Methods Review of antiretroviral technical datasheets, pegylated interferon, ribavirin and protease inhibitors (boceprevir/telaprevir) and of the necessary literature to collect criteria and general recommendations for treatment of these diseases, adverse drug effects, interactions between these drugs and others, and contraindications for use.

Results In order to implement SiMON in the pharmaceutical monitoring of patients with $\mathrm{HIV} / \mathrm{HCV}$, the Pharmacy Service reviewed 15 datasheets of antiviral drugs. Usage alerts were established as well as recommendations for each drug that depend on patient data (83 alerts), prescribed dosage ( 34 alerts), laboratory test results $(94$ alerts) and interactions between different medicinal products (484 alerts). Each of these alerts can refer to a contraindication or usage precaution, with a possible recommendation to suspend treatment, adjust the dose or change the drug involved in the interaction for an alternative. We also collected 482 adverse drug effects that had to be structured in tree form so they could be encoded by the Computing Service.

Conclusions The SiMON protocol, a tool that increases the efficiency of patient monitoring in a multidisciplinary way, makes it possible to record side effects and generate drug alerts and it may be possible to make additional use of the data stored. Collaboration between different services increases the performance of tools at our disposal.

No conflict of interest. 